\title{
GAMMA-RAY BURST LIGHT CURVES IN THE RELATIVISTIC TURBULENCE AND RELATIVISTIC SUBJET MODELS
}

\author{
AYAH LAZAR ${ }^{1,2}$, EHUd NAKAR ${ }^{3,4}$, AND TSVI PIRAN ${ }^{1}$ \\ ${ }^{1}$ The Racah Institute of Physics, Hebrew University, Jerusalem 91904, Israel \\ 2 Department of Geophysics and Planetary Sciences, Tel Aviv University, Tel Aviv 69978, Israel \\ ${ }^{3}$ Raymond and Beverly Sackler School of Physics \& Astronomy, Tel Aviv University, Tel Aviv 69978, Israel \\ ${ }^{4}$ Theoretical Astrophysics, Caltech, Pasadena, CA 91125, USA \\ Received 2008 December 23; accepted 2009 February 18; published 2009 March 18
}

\begin{abstract}
Randomly oriented relativistic emitters in a relativistically expanding shell provide an alternative to internal shocks as a mechanism for producing gamma-ray bursts' variable light curves with efficient conversion of energy to radiation. In this model, the relativistic outflow is broken into small emitters moving relativistically in the outflow's rest frame. Variability arises because an observer sees an emitter only when its velocity points toward him so that only a small fraction of the emitters is seen by a given observer. Significant relativistic random motion requires that a large fraction of the overall energy is converted to random kinetic energy and is maintained in this form. While it is not clear how this is achieved, we explore here, using two toy models, the constraints on parameters required to produce light curves comparable to the observations. We find that a tight relation between the size of the emitters, and the bulk and random Lorentz factors is needed and that the random Lorentz factor determines the variability. While both models successfully produce the observed variability there are several inconsistencies with other properties of the light curves. Most of which, but not all, might be resolved if the central engine is active for a long time, producing a number of shells, resembling to some extent the internal shocks model.
\end{abstract}

Key words: gamma rays: bursts - turbulence

\section{INTRODUCTION}

Gamma-ray bursts (GRBs) temporal variability played a major role in the understanding how GRBs operate. Standard external shocks, in which the external medium slows the relativistic ejecta, cannot produce efficiently variable light curves (Sari \& Piran 1997). While internal shocks resolve the variability and agree with other properties of GRB light curves (e.g., Nakar \& Piran 2002b; Ramirez-Ruiz \& Fenimore 2000) they suffer from several drawbacks. First and foremost is their low efficiency (Kobayashi et al. 1997; Daigne \& Mochkovitch 1998; see, however, Kobayashi et al. 1997; Kobayashi \& Sari 2001; Beloborodov 2000). This is particularly troublesome in view of the high efficiency implied from the comparison of the prompt $\gamma$-rays luminosity and the kinetic energy that remains in the outflow. Detailed models for the emission mechanisms of the prompt $\gamma$-rays pose other problems (Kumar \& McMahon 2008).

External shocks can produce highly variable light curves if the outflow is slowed down by small external clumps; each clump producing a short pulse. However, this process will inevitably be inefficient (Sari \& Piran 1997) as the overall covering factor of the emitting regions is $\delta t / T$ ( $\delta t$ and $T$ are the pulses' and the burst's durations). Observed values of $\delta t / T$ are typically $\sim 0.01$ and it can be as low as $10^{-4}$ (Nakar \& Piran 2002a).

Lyutikov \& Blandford (2002, 2003) (see, also, Lazar 2005 [L05]; Lyutikov 2006; Narayan \& Kumar 2009) proposed that variability can be recovered while maintaining high efficiency if the shell that moves with a bulk Lorentz factor $\Gamma$ contains emitting clumps (see Figure 1) that move with random macroscopic relativistic velocities (with a Lorentz factor $\gamma^{\prime}$ ). A clump is observed only when its radiation cone (with an opening angle of the order of $1 / \Gamma \gamma^{\prime}$ in the lab frame) points toward the observer. The filling factor of the clumps may be unity, recovering high efficiency. However, as only a small fraction of the clumps are observed at any given time, the light curve can show rapid variability. The overall duration of the burst, is larger between the angular time and the shell's light crossing time $\left(\max \left\{R / c \Gamma^{2}, \Delta / c\right\}\right)$, where $R$ and $\Delta$ are the shell's radius and width, respectively, and $c$ is the light speed, allowing for emission radii much larger than $\delta t \Gamma^{2}$, as required in the internal shocks model. The temporal variability is then dictated by the random Lorentz factor, $\gamma^{\prime}$, reflecting the activity of the emitting region and not those of the inner engine.

While it is unclear how macroscopic random relativistic motion can be generated, we assume that it does and examine, using two simplified toy models that include the essential ingredients, the conditions under which the temporal features of the observed light curve can be produced (see L05). We describe our first toy model, which we call here relativistic turbulence, and derive analytic constraints and numerical light curves in Section 2. In Section 3, we consider a second toy model proposed by Lyutikov (2006) that is based on subjets (SJs) and compare it with the first one. We summarize the results and compare both models with observations in Section 4.

\section{RELATIVISTIC TURBULENCE}

Our (L05) kinematic toy model for relativistic turbulence considers a shell which is divided into discrete randomly distributed emitters that have randomly oriented relativistic velocities. The emitters change their direction of motion continuously, as expected in a turbulent medium. Since the emitters exhibit a coherent macroscopic motion, we require that each emitter be causally connected, and that it changes its direction on times longer than the causal timescale but shorter than the shell crossing time. The length scale of the emitter in its own rest frame, $l^{\prime \prime}$, is assumed to be similar in all three dimensions, and it emits isotropically in this frame. We define a dimensionless scale $\psi \equiv l^{\prime \prime} / R$, which is the angular scale of an emitter that points toward the observer (see inset of Figure 1). The emitters radiate as the shell moves 


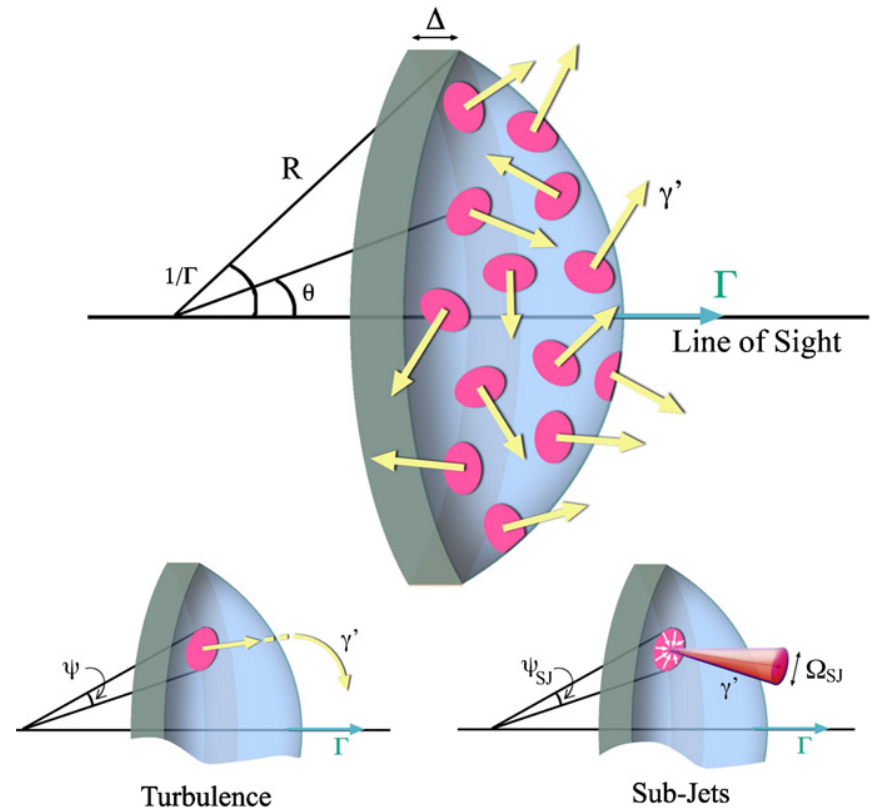

Figure 1. Basic kinematic model-relativistically expanding shell with an ensemble of emitters that are moving at random relativistic velocities within the shell. The inserts describe the geometrical details and definitions of the two alternative models. Left: relativistic turbulence-emitters of size $R \psi$ move relativistically. Right: SJs—energy from regions of size $R \psi_{\mathrm{SJ}}$ is extracted into relativistic jets.

from $R_{0}$ to $2 R_{0}$. Due to the turbulent motion the positions and directions of the emitters change with time. We model this by a set of successive shells between $R_{0}$ and $2 R_{0}$. Each new shell is constructed with randomly distributed emitters, representing the random changes in direction of the turbulent motion. The time difference between two shells is, $\tau^{\prime}$, the time it takes for the emitters to turn an angle of $\gamma^{\prime-1}$ (in the shell frame).

Note that there are three frames: the lab frame; the shell's frame, denoted by a prime, which is boosted radially with a Lorentz factor $\Gamma$ relative to the lab; and the frame of each emitter, denoted by two primes, which is boosted by (randomly oriented) $\gamma^{\prime}$ relative to the shell frame. The observer is, of course, at rest relative to the lab frame. However, the observer time, namely the arrival time of photons (denoted $t$ ) differs from the lab time by the usual time of flight arguments (Rybicki \& Lightman 1979).

The Doppler shift from an emitter is

$$
\Lambda=[\gamma(1-\beta \cdot \cos \alpha)]^{-1},
$$

where $\gamma, \beta$, and $\alpha$ are the Lorentz factor, velocity and the angle between the velocity and the line to the observer (both in the lab frame). The flux that reaches the observer from this emitter is

$$
F_{v}=\int I_{v^{\prime \prime}}^{\prime \prime} \Lambda^{3} d \Omega_{i} \approx I_{v^{\prime \prime}}^{\prime \prime} \Lambda^{3} \frac{\psi^{2} R^{2}}{D^{2}},
$$

where $I_{v^{\prime \prime}}^{\prime \prime}$ is the specific intensity and the second relation holds for a small enough emitter ( $D$ is the distance to the observer). An implicit $K$-correction arises from the difference between the $v$ and $v^{\prime \prime}$.

Define $\theta$ as the angle between the line that connects the origin and the emitter and the line that connects the origin and the observer (see inset of Figure 1). The maximal Doppler boost, $\Lambda_{\max }=4 \gamma^{\prime} \Gamma$, is obtained for emitter at $\theta=0$ that moves along the line of sight. The flux decreases like $\Lambda^{3}$ plus a $K$-correction. Therefore, we consider emitters only if
$\Lambda_{i}>\Lambda_{\max } / 2$. What then is the probability that an emitter at angle $\theta$ is observed, $d P / d \theta \equiv S\left(\theta, \Gamma, \gamma^{\prime}\right)$ ? At $\theta=0$ it is $S\left(0, \Gamma, \gamma^{\prime}\right) \approx 1 / 4 \gamma^{\prime 2}$ while $S\left(1 / \Gamma, \Gamma, \gamma^{\prime}\right)=0$. This suggests that $S$ scales as $S\left(\theta, \Gamma, \gamma^{\prime}\right)=\gamma^{\prime-2} \tilde{S}(\theta \Gamma)$ (L05), implying that the average probability that an emitter will be visible from an arbitrary position on the shell is

$$
P\left(\Gamma, \gamma^{\prime}\right) \approx \frac{1}{2\left(\Gamma \gamma^{\prime}\right)^{2}} \int_{0}^{1} \tilde{S}(\theta \Gamma)(\theta \Gamma) d(\theta \Gamma) \approx \frac{0.3}{4 \pi\left(\Gamma \gamma^{\prime}\right)^{2}} .
$$

The factor 0.3 was evaluated numerically (L05) and can be ignored at the accuracy level of our discussion.

The arrival time from an emitter at $R, \theta$ is

$$
T=\frac{R-R_{0}}{2 c \Gamma^{2}}+\frac{R \theta^{2}}{2 c}+\frac{x}{c},
$$

where $x(<\Delta)$ is the distance of the emitter from the front of the shell. As the last photons will arrive from $2 R_{0}$, an angle of $1 / \Gamma$ and $x=\Delta$, the overall duration of the burst will be a function only of $\Delta$ and $\Gamma$ (and not $\left.\gamma^{\prime}\right)$ :

$$
T \approx \frac{R_{0} d}{c \Gamma^{2}},
$$

where we define $d \equiv \Delta \Gamma^{2} / R$. As the shell is expected to expand relativistically in its own frame $d \gtrsim 1$. For $d>1$, the shell's width, as well as $T$, is determined by the engine activity time while for $d=1$ they do not.

The duration of a pulse arriving from a single emitter is the longest of the three following timescales.

1. The duration over which the emitter points toward the observer, namely the duration over which the direction of motion varies by an angle $1 / \Gamma \gamma^{\prime}$ in the lab frame $\left(1 / \gamma^{\prime}\right.$ in the shell's frame). As the emitter is confined to the shell it should make at least a $\pi / 2$ turn during $\Delta^{\prime} / c$, implying that the time to turn by $1 / \gamma^{\prime}$ (shell's frame), $\tau^{\prime}$, is shorter than $\Delta^{\prime} / c \gamma^{\prime}$. Causality puts a lower limit on $\tau^{\prime}$ of $R \psi / c$. Therefore,

$$
R \psi / c \leqslant \tau^{\prime} \leqslant \Delta^{\prime} / c \gamma^{\prime}
$$

In the observer's frame, this translates to

$$
R \psi / \Gamma \gamma^{\prime 2} c \leqslant \tau \leqslant \Delta / c \gamma^{\prime 3} .
$$

2. The emitter's light crossing time in the lab frame (in the direction along the line of sight). For an emitter moving toward the observer this time is $R \psi / \gamma^{\prime} \Gamma$.

3. The angular timescale — at the largest possible angle, where the emitter is still visible by the observer, $1 / \gamma^{\prime} \Gamma$, the time difference between the first and the last photon would be $\frac{1}{c} R \psi \sin \left(1 / \gamma^{\prime} \Gamma\right) \approx R \psi / c \gamma^{\prime} \Gamma$. Overall (2) and (3) are of the same order and much larger than (1). Thus,

$$
\delta t \approx R \psi / c \gamma^{\prime} \Gamma .
$$

Using Equations (5) and (8) we express, $N_{p}$, the number of (possibly overlapping) pulses expected in a burst:

$$
N_{p} \equiv n_{p} \frac{T}{\delta t}=n_{p} \frac{d \gamma^{\prime}}{\psi \Gamma},
$$

\footnotetext{
5 Note that for a hydrodynamic external shock $d \lesssim 1$ (Sari \& Piran 1997) but this might not be relevant here.
} 
where $n_{p}$ is the occupation number of pulses at any given observer time (i.e., $n_{p} \gg 1$ implies many overlapping pulses while $n_{p} \ll 1$ implies long quiescent periods between isolated pulses).

The number of emitters is $4 \pi R^{2} \Delta^{\prime} /(R \psi)^{3}=4 \pi \Delta \Gamma / R \psi^{3}$. The emitters obtain new random directions (which differ by more than $1 / \gamma^{\prime}$, in the shell's frame, than the previous ones) after a time $\tau^{\prime}$. Thus, the total number of independent emitters, $N_{\text {tot }}$, is larger by the factor $R /\left(c \Gamma \tau^{\prime}\right)$; the ratio of the total duration over which the radius doubles and $\tau^{\prime}$. Finally we introduce a filling factor $f \leqslant 1$ allowing for the possibility that not all emitters are active all the time or that space is not fully covered by emitters ( $f \ll 1$ is strongly disfavored as the efficiency is always smaller than $f$ ). Overall, we find

$$
N_{\mathrm{tot}}=\frac{4 \pi f}{\psi^{3}} \frac{d}{\Gamma^{2}} \frac{R}{c \tau^{\prime}} .
$$

The condition $N_{P}=P N_{\text {tot }}$ yields

$$
n_{p}=\frac{f d}{\gamma^{\prime 3} \Gamma^{3} \psi^{2}} \frac{R}{c \tau^{\prime}},
$$

and using (7)

$$
\frac{f}{d\left(\gamma^{\prime} \Gamma \psi\right)^{2}} \leqslant n_{p} \leqslant \frac{f}{\left(\gamma^{\prime} \Gamma \psi\right)^{3}} .
$$

We demand $n_{p} \approx 1$ since many overlapping pulses reduce the observed variability, whereas very frequent long quiescent times between pulses are not observed. If the shell is in the freely expanding phase (i.e., $d \approx 1$ ) $n_{p}$ will be of order unity if

$$
\psi \approx f^{1 / k} \frac{1}{\gamma^{\prime} \Gamma}
$$

where $k$ is between 2 and 3. Narayan \& Kumar (2009) have pointed out that $\psi=1 / \gamma^{\prime} \Gamma$ if one requires that the emitters be of the maximal causally allowed size. Note that $n_{p}$ depends quite sensitively on $\gamma^{\prime} \Gamma \psi$ and it increases rapidly if $\psi$ is smaller than $1 / \gamma^{\prime} \Gamma$. This implies, for example, that a significant number of small eddies, which may arise in a turbulent cascade, may be problematic. Using the relations (13) and (9), and assuming the causal limit for $\tau^{\prime}$

$$
\gamma^{\prime} \approx\left(\frac{f}{n_{p}}\right)^{1 / 6} \sqrt{\frac{T}{d \delta t}},
$$

leading to $\gamma^{\prime} \approx 10 / \sqrt{d}$ for typical values of $T / \delta t$. Note that while the model determines $\gamma^{\prime}$ it does not constrain $\Gamma$ and $R$.

Figure 2 depicts simulated light curves (L05) for four choices of parameters. The two upper panels have $n_{p}=1$ with different emitter sizes. Both light curves are highly variable and densely filled with nonoverlapping pulses. However, as $d=1$, the underlying overall envelope of the pulses is seen. As the emitters are smaller on the right panel there are more pulses than the left one. The envelope is observed since only a small fraction of the volume and hence fewer pulses are seen early on. Similarly at $t>(d+1) R_{0} / 2 c \Gamma^{2}$ pulses from small $\theta$ values are not seen, implying that only lower amplitude pulses (on average) are observed during the last $T /(d+1)$ of the burst. The envelope is stretched on the bottom panels where $d=10$. The lower left panel depicts a very low $n_{p}$ with a rather sparse light curve. The lower right panel depicts a light curve of a wide shell and $n_{p}=0.7$, which is rather similar to observed bursts. For $n_{p} \gg 1$ (not shown) the pulses are overlapping and all variability is erased, leaving only the envelope.

\section{RELATIVISTIC SUBJETS}

Motivated by reconnection in highly magnetized outflow, Lyutikov (2006) considered a model in which relativistic SJs are accelerated to a Lorentz factor $\gamma^{\prime}$ by dissipation of the bulk energy in many different "mini-engines" within the relativistically expanding shell. These "mini-engines" or acceleration sites correspond to reconnection sites within the magnetized flow (e.g., Lyutikov \& Blackman 2001). The "mini-engines" are at rest in the shell frame. Each mini-engine operates for a time $t_{\mathrm{SJ}}^{\prime}$. The directions of the accelerated SJs are random in the shell frame but the opening angle and direction of each is constant while its mini-engine is active.

The SJ extracts energy from a region of size $l^{\prime}=\delta t_{\mathrm{SJ}}^{\prime} \hat{\beta} c$, where $\hat{\beta} c$ is the speed of extraction of energy from the surrounding region (relativistic reconnection suggests $\hat{\beta} \approx 0.1$, Lyubarsky 2005). The observed duration is

$$
\delta t_{\mathrm{SJ}}=\frac{t_{\mathrm{SJ}}^{\prime}}{\Gamma} \approx \frac{R \psi_{\mathrm{SJ}}}{\hat{\beta} c \Gamma},
$$

where we define the dimensionless parameter $\psi_{\mathrm{SJ}} \equiv l^{\prime} / R$. Slightly generalizing Lyutikov (2006) we write the probability to observe an emitter as $\phi^{2} / 4 \pi \Gamma^{2}$, where $\phi=\max \left(\sqrt{\Omega_{\mathrm{SJ}}}, 1 / \gamma^{\prime}\right)$ and $\Omega_{\mathrm{SJ}}$ is the SJ opening solid angle. Following the notation of Section 2, we define, $f$, the filling factor of regions from which energy is extracted into the SJs $\left(N_{\text {tot }} \equiv f 4 \pi \Delta^{\prime} R^{2} / l^{\prime 3}\right)$. The occupation number of observed pulses is

$$
n_{p_{\mathrm{SJ}}}=\frac{f \phi^{2}}{\hat{\beta} \psi_{\mathrm{SJ}}^{2} \Gamma^{2}} .
$$

The condition $n_{p_{\mathrm{SJ}}} \approx 1$ yields

$$
\psi_{\mathrm{SJ}} \approx \frac{\sqrt{f} \phi}{\hat{\beta}^{1 / 2} \Gamma},
$$

and

$$
\frac{T}{\delta t} \approx \frac{\hat{\beta}^{3 / 2} d}{\phi \sqrt{f}} \leqslant \gamma^{\prime} \frac{\hat{\beta}^{3 / 2} d}{\sqrt{f}} .
$$

This implies that an efficient $(f \sim 1)$ highly variable burst requires either a large $\gamma^{\prime}$ or a wide shell (for $T / \delta t \sim$ $100, \gamma^{\prime} d \sim 100 \hat{\beta}^{-3 / 2}$.).

This constant direction of the emitters and the fact that causality in the shell's frame determines the SJ size, $c \delta t^{\prime}$, are the main kinematic differences between the SJ and the turbulence model (in which the emitter's direction varies and causality in the emitter's frame determines its size, $\left.l^{\prime \prime}\right)$. For the same $n_{p}$ and $\delta t / T$ the two models give similar light curves. In particular, an overall (rising and falling) envelope for the light curve is expected in the SJ model as well.

\section{DISCUSSION AND CONCLUSIONS}

We have derived conditions on the parameters of relativistic random emitters needed for producing variable GRB light curves. This is characterized by $n_{p} \approx 1$ which ensures that typical pulses do not overlap and are not too sparse either. Our numerical simulations show that for $0.03<n_{p}<3$ one obtains light curves that resemble observed GRBs (see Figure 2). The resulting light curves do not change qualitatively when we introduce a distribution of turbulent Lorentz factors and sizes. 

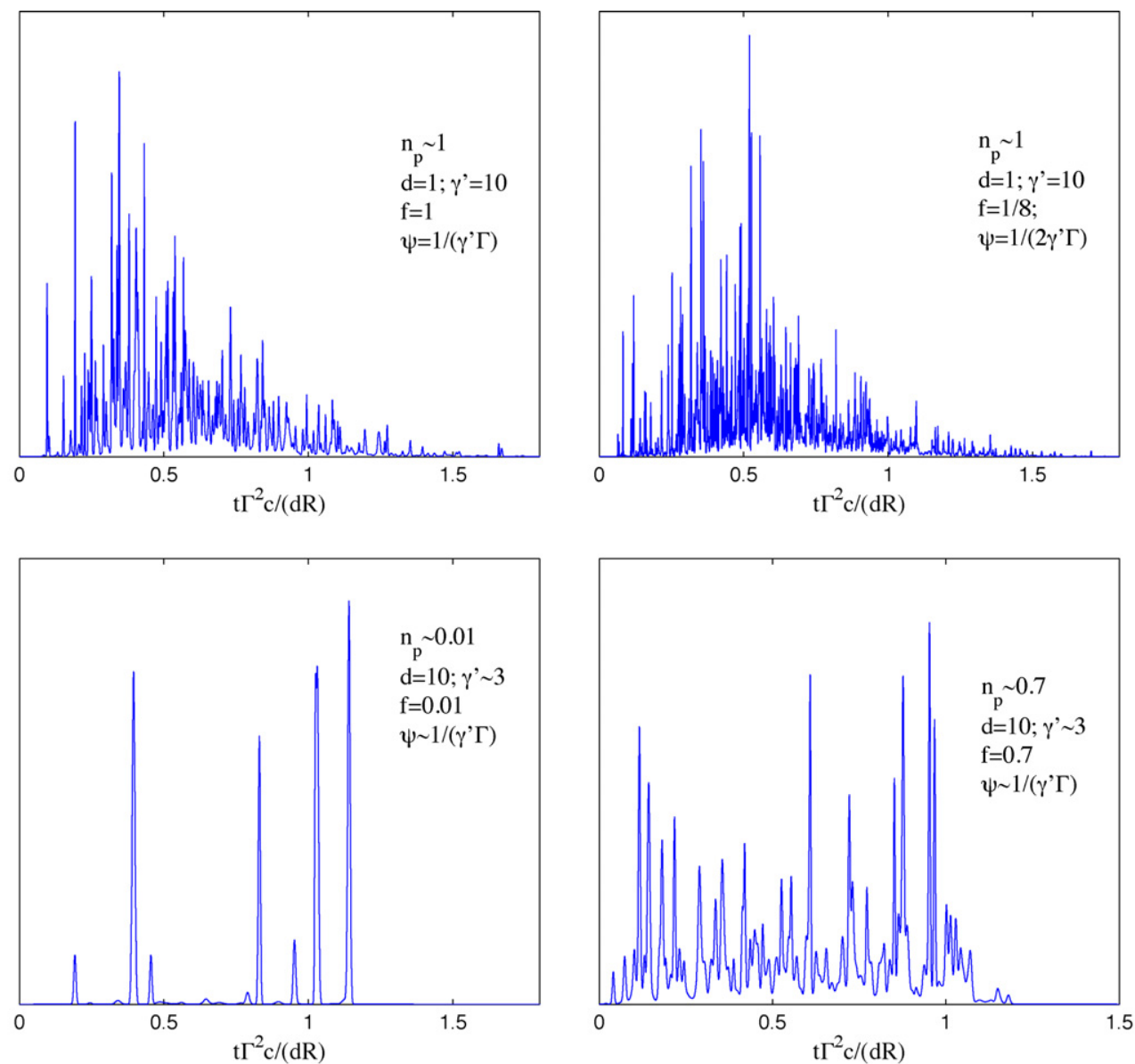

Figure 2. Numerical Monte Carlo simulations of light curves for different combinations of parameters (shown on each frame; the scalings used eliminate the dependence on $R$ and $\Gamma$ ). The flux of each pulse is calculated assuming that the radiation efficiency is constant per unit mass for all emitters in their rest frame, namely that $I_{v^{\prime \prime}}^{\prime \prime} \propto(\psi R)^{-3}$, therefore $F_{v} \propto \Lambda^{3} /(\psi R)$. We approximate each pulse as a Gaussian with the above parameters and we sum over all contributions to construct a light curve. In both upper frames $n_{p}=1$ and $d=1$. In the top left, the emitters are as large as causality allows while on the top right they are half of this value and the filling factor is lowered to compensate. In both the overall envelope is clearly seen. The sparsity of pulses is apparent when $n_{p}=0.01$ (bottom left) and the "straightening out" of the envelope is clearly seen (bottom two panels) when $d=10$.

Causality suggests, for relativistic turbulence, that the relation $\psi=1 / \Gamma \gamma^{\prime}$ between the angular size of the emitters, $\psi$, and the turbulent and the bulk Lorentz factors holds naturally (Narayan \& Kumar 2009). But, this condition holds when the turbulent eddies are of the maximal possible size and may be broken by cascade to lower scales. The condition, $\psi_{\mathrm{SJ}}=\sqrt{f} \phi / \hat{\beta}^{3 / 2} \Gamma$ arises in the SJs model. For high efficiency, negligible SJ opening angle assuming $\hat{\beta} \sim 1$ this reduces to $\psi_{\mathrm{SJ}} \sim 1 / \Gamma \gamma^{\prime}$ or $t_{\mathrm{SJ}}^{\prime} \approx R / c \Gamma \gamma^{\prime}$. While this is similar to the one obtained in the turbulent model, here there is no apparent physical motivation for proportionality between $t_{\mathrm{SJ}}^{\prime}$ and $1 / \gamma^{\prime}$ and this requires an ad hoc fine tuning.

In both models the light curves arising from a single expanding shell with $d \approx 1$ shows a rising and falling underlying envelope. Furthermore, a single shell cannot produce bursts which depict long quiescent periods. The envelope can be erased if $d \gg 1$, while quiescent periods require an outflow of several shells (where naturally $d \gg 1$ ). These solutions become marginal in the turbulent model if $\tau^{\prime}$ is determined by causality, since $\gamma^{\prime} \gg 1$ requires $d \lesssim 10$ (see Equation (14)). The SJ model, however, may favor $d \gg 1$ as it reduces the required value of $\gamma^{\prime}$.

It seems that with proper conditions (and rather reasonable in the case of the relativistic turbulence) these models can produce (efficiently) the observed highly variable GRB light curves.
We turn now to several shortcomings. First and foremost is the question how such macroscopic relativistic motions can be generated and sustained. One needs to convert $\sim\left(1-1 / \gamma^{\prime}\right) f$ of the initial total energy to the kinetic energy of the emitters and further dissipation in the emitters' frame is needed to generate the radiation. Additional questions involve the shape and other properties of individual pulses versus those seen in observed pulses: (1) GRBs show a clear difference between the fast rise and the slow decline of individual pulses (Norris et al. 1996). The light curve of an individual pulse results from a combination of the motion of the emitter, its orientation relative to the observer, its width as well as intrinsic inhomogeneities within the emitter. In the relativistic turbulence model the emitter was radiating long before its velocity pointed toward the observer and it continues to emit long after it moves away from the observer. There is no reason (on average) for a difference between the rising and falling phases of an individual pulse. ${ }^{6}$ This is not a problem in the SJs model in which the onset of the pulse corresponds to the beginning of the activity of the emitter. (2) The temporal structure of the first and second halves of GRB

\footnotetext{
6 Note that systematic variation of the emitter properties on a timescale of $\tau^{\prime}$ will result in a strong signature differentiating between early and late phases of the overall light curve, which is not observed. On the other hand, nonsystematic variations (e.g., deceleration and acceleration) are expected to result in similar affects on the temporal structure of rising and decaying parts of pulses.
} 
light curves are similar (Ramirez-Ruiz \& Fenimore 2000). The light curves produced in the two models have an overall envelope that favors stronger pulses earlier and weaker ones later. This might be resolved by a combination of several emitting shells or with very wide shells, but here fine tuning is required in the turbulent model in order to keep $\gamma^{\prime} \gg 1$. (3) Weaker and denser pulses (arriving from emitters not moving directly toward the observer) continue at $t>T$ producing the typical envelope of high-latitude emission (Kumar \& Panaitescu 2000). This is consistent with rapid declines seen in some of the early afterglows. However, in many cases the decline is faster. In the internal shocks model this is attributed to the dominant contribution of a late pulse that shifts the zero point of the time. Such an option does not arise here unless, once more, we allow for several shells or a single wide shell. (4) The duration of an observed pulse is correlated with the preceding interval (Nakar \& Piran 2002b; Quilligan et al. 2002). There is no reason that such correlation should appear in both models. (5) These models predict a Doppler-induced correlation between the intensity and $E_{\text {peak }}$. While stronger peaks are typically harder, it is not clear whether this specific relationship is satisfied.

We could not find obvious modifications that will address all these issues. While it is not clear that those cannot be found, this suggests that the simple versions of these models might not be enough. A simple extension of a wide shell $d \gg 1$ or several separated shells might resolve some of the issues and it might be essential for the SJ model allowing moderate values of the SJs Lorentz factor.
This research is supported by the ISF center of excellence in High Energy Astrophysics (T.P. and A.L.), Marie Curie IRG grant (E.N.), advanced ERC excellence award and the Schwartzmann chair (T.P.).

\section{REFERENCES}

Beloborodov, A. M. 2000, ApJ, 539, L25

Daigne, F., \& Mochkovitch, R. 1998, MNRAS, 296, 275

Kobayashi, S., Piran, T., \& Sari, R. 1997, ApJ, 490, 92

Kobayashi, S., \& Sari, R. 2001, ApJ, 551, 934

Kumar, P., \& McMahon, E. 2008, MNRAS, 384, 33

Kumar, P., \& Panaitescu, A. 2000, ApJ, 541, L51

Lazar, A. 2005, MSc. Thesis, Kinematics of Time-Scale Variability in Gamma-

Ray Bursts: The Relativistic Turbulence Model, Hebrew Univ., L05

Lyubarsky, Y. E. 2005, MNRAS, 358, 113

Lyutikov, M. 2006, MNRAS, 369, L5

Lyutikov, M., \& Blackman, E. G. 2001, MNRAS, 321, 177

Lyutikov, M., \& Blandford, R. 2002, arXiv:astro-ph/0210671

Lyutikov, M., \& Blandford, R. 2003, arXiv:astro-ph/0312347

Nakar, E., \& Piran, T. 2002a, MNRAS, 330, 920

Nakar, E., \& Piran, T. 2002b, MNRAS, 331, 40

Nakar, E., \& Piran, T. 2002c, ApJ, 572, L139

Narayan, R., \& Kumar, P. 2009, MNRAS, 394, L117

Norris, J. P., et al. 1996, ApJ, 459, 393

Quilligan, F., McBreen, B., Hanlon, L., McBreen, S., Hurley, K. J., \& Watson, D. $2002, A \& A, 385,377$

Ramirez-Ruiz, E., \& Fenimore, E. E. 2000, ApJ, 539, 712

Rybicki, G. B., \& Lightman, A. P. 1979, Radiative Processes in Astrophysics (New York: Wiley)

Sari, R., \& Piran, T. 1995, ApJ, 455, L143

Sari, R., \& Piran, T. 1997, ApJ, 485, 270 